\title{
Herramienta para la enseñanza de la lengua Mazateca basada en Realidad Aumentada
}

\section{Tool for the teaching of the Mazatec language based on Augmented Reality}

MOTA-CARRERA, Luis Cresenciot, MÁRQUEZ-DOMÍNGUEZ*, José Alberto, SABINO-MOXO, Beatriz Adriana y SÁNCHEZ-ACEVEDO, Miguel Ángel

Universidad de la Cañada (UNCA), Oaxaca, México

ID $1^{\mathrm{er}}$ Autor: Luis Cresencio, Mota-Carrera / ORC ID: 0000-0001-9427-5002, CVU CONACYT ID: 999991

ID 1 $1^{\mathrm{er}}$ Coautor: José Alberto, Márquez-Domínguez / ORC ID: 0000-0003-2552-2289, CVU CONACYT ID: 210472

ID $2^{\text {do }}$ Coautor: Beatriz Adriana, Sabino-Moxo / ORC ID: 0000-0002-8577-494X, CVU CONACYT ID: 210495

ID3 ${ }^{\text {er }}$ Coautor: Miguel Ángel, Sánchez-Acevedo / ORC ID: 0000-0002-0996-0038, CVU CONACYT ID: 205720

\section{Resumen}

En este trabajo se presenta una herramienta para la enseñanza de la lengua mazateca basado en la tecnología de Realidad Aumentada (RA), dirigido a los profesores del centro de educación preescolar indígena Naxhó Café, ubicada en la población de Huautla de Jiménez, Oaxaca. El proyecto fue desarrollado con la metodología de Desarrollo Centrado en el Usuario (DCU), esta metodología permitió llevar a cabo dos actividades de enseñanza con alumnos de primer, segundo y tercer año de preescolar obteniendo resultados considerables en la eficiencia, eficacia y satisfacción de dicha herramienta.

\section{Mazateca, UCD, Realidad aumentada}

\begin{abstract}
This paper presents a tool for teaching the Mazatec language based on Augmented Reality (RA) technology, aimed at teachers at the Naxhó Café indigenous preschool education center, located in the town of Huautla de Jiménez, Oaxaca. The project was developed using the methodology of User Center Desing (DCU), this methodology allowed to carry out two teaching activities with students of first, second and third year of preschool with which they could obtain considerable results in efficiency, efficiency and satisfaction of said tool.
\end{abstract}

Mazatec, UCD, Augmented Reality Aumentada. Revista de Cómputo Aplicado. 2019, 3-11: 8-15

\footnotetext{
* Correspondencia al Autor (Correo electrónico: albertomarquez@unca.edu.mx)

$\dagger$ Investigador contribuyendo como primer Autor.
} 


\section{Introducción}

Lengua materna, también conocida como primera lengua es el que una persona aprende y adquiere en primera instancia dentro del entorno inmediato en el cual crece y se desenvuelve (Galdames et al., 2006).

Datos del INALI (Instituto Nacional de Lenguas Indígenas) proporciona información del grado de desaparición de la lengua Mazateca, en el año 2010 había $20.3 \%$ de hablantes mazatecos y en el 2015 se redujo al $15.6 \%$, es por ello la importancia de establecer herramientas educativas que apoyen en la conservación, difusión y promoción de las lenguas maternas.

De acuerdo a la Secretaria de Cultura de México, hasta el año 2018 se contemplaban registros de 7,000 idiomas alrededor del mundo, de los cuales, casi el 50 por ciento se encuentra en peligro de desaparecer. México ocupa un segundo lugar entre las primeras 10 naciones de América Latina con mayor riqueza y diversidad lingüística contando con 69 lenguas nacionales 68 indígenas y el español- (Secretaría de Cultura, 2018).

Tal como lo señala El Programa Especial de los Pueblos Indígenas (2014-2018), las comunidades hablantes de alguna lengua indígena se enfrentan a serios problemas tales como la discriminación, estigmatización, migración, globalización, poco o nulo involucramiento de las instituciones educativas para fomentar la conservación de las lenguas, entre otros, estos factores influyen en la pérdida directa o indirecta de las lenguas.

Ante este fenómeno, se vio una oportunidad para contribuir en el rescate de la lengua materna mazateca variante de Huautla de Jiménez, Oaxaca. En coordinación con profesores del centro indígena de educación preescolar bilingüe Naxhó Café se logró el desarrollo de materiales didácticos de enseñanza y una aplicación para dispositivos móviles que integra tecnología de realidad aumentada, con el objetivo de poner en sus manos una herramienta tecnológica para complementar el trabajo que realizan a través de la enseñanza del mazateco.

\section{Desarrollo}

A continuación, se describen los principales aspectos en el desarrollo de la herramienta para la enseñanza de la lengua materna mazateca.

\section{Lengua materna mazateca de Huautla de Jiménez, Oaxaca}

La lengua mazateca en el estado de Oaxaca se habla en municipios y localidades que se encuentran en las regiones de la montaña en la parte alta, región de la Cañada, y de la zona baja que corresponde a la región de la Cuenca del Papaloapan (Guzmán, 2011).

Los mazatecos se autodenominan $\mathrm{Ha}$ shuta Enima, que en su lengua quiere decir "los que trabajamos el monte, humildes, gente de costumbre". Según otros autores, el origen del nombre mazateco viene del náhuatl mazatecatl, o "gente del venado", nombre que les fue dado por los nonoalcas debido al gran respeto que tenían por el venado (INPI, 2017).

\section{Las TIC en el rescate de las lenguas maternas}

Tal como lo señala la UNESCO, las tecnologías de la información y comunicación se consideran factores estratégicos para ampliar la funcionalidad de las lenguas indígenas, ya que permiten potenciar su uso en diferentes entornos y su aprovechamiento puede ser dirigido al desarrollo social, académico, institucional y comunicacional (PROINALI, 2014).

Dentro de las TIC se encuentra la realidad aumentada, aquella tecnología que permite percibir, ver y conectarnos con el mundo mediante el uso de dispositivos tecnológicos interactuando con un entorno virtual, donde es posible añadir al mundo físico información virtual tales como, objetos tridimensionales, video, audio, texto el cual permite explotar una gran variedad de beneficios (UNESCO, 2016).

Gracias al software libre existe una multitud de herramientas que permiten integrar tecnología de realidad aumentada en aplicaciones móviles. Para el trabajo a desarrollar se eligieron las siguientes: 


\section{Pixabay.com}

Es un sitio web con una comunidad de creativos que comparten imágenes sin derechos de autor. Todos los contenidos se publican bajo la Licencia Pixabay, que los hace seguros para usar sin pedir permiso o dar crédito al artista, incluso con fines comerciales.

\section{Poly de Google}

Poly es una biblioteca en línea donde las personas pueden navegar, compartir y mezclar activos 3D. Un activo es un modelo 3D o una escena creada con Tilt Brush, Blocks o cualquier programa 3D que genere un archivo que se pueda cargar en Poly. Muchos activos están autorizados bajo la licencia CC BY, lo que significa que los desarrolladores pueden usarlos en sus aplicaciones de forma gratuita, siempre que el creador reciba crédito (Poly-Google, 2019).

\section{Blender}

Es el paquete de creación 3D de código abierto y gratuito. Admite la totalidad de la canalización 3D: modelado, rigging, animación, simulación, renderización, composición y seguimiento de movimiento, incluso edición de video y creación de juegos (Blender, 2019).

\section{Vuforia Engine}

Es la plataforma más utilizada para el desarrollo de RA, con soporte para teléfonos, tabletas y gafas. Cuenta con SDK (Kit de Desarrollo de Software) que permite construir aplicaciones basadas en realidad aumentada (Vuforia Engine, 2019).

\section{Unity 3D}

Es una de las plataformas más amplias para el desarrollo de videojuegos siendo uno de los líderes en el manejo de tecnología de RA para dispositivos móviles (Unity 3D, 2019).

A continuación, se propone el desarrollo de la herramienta.

\section{Desarrollo de la Herramienta}

Para el desarrollo del proyecto se siguió la metodología de Diseño Centrado en el Usuario (DCU).

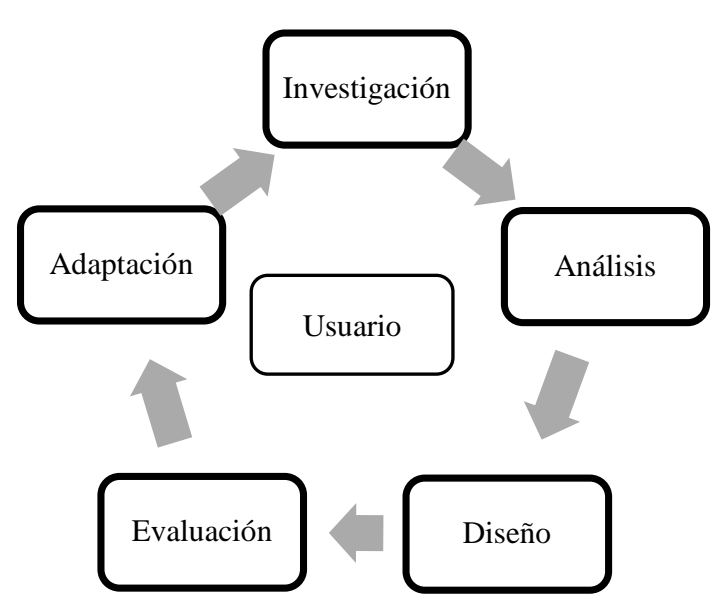

Figura 1 Etapas del Diseño Centrado en el Usuario. Ciclo Iterativo

Fuente: Garreta y Mor (2019)

Como lo indican los autores Garreta y Mor (2019), el objetivo del DCU es la creación de productos que los usuarios encuentren útiles y usables; es decir, que satisfagan sus necesidades teniendo en cuenta sus características. Empleando la metodología (Figura 1) permitió conocer y comprender las necesidades, limitaciones, comportamientos y características que tienen los profesores y alumnos del preescolar, para llevar a cabo la herramienta acorde a las necesidades específicas, involucrándolos en todo el proceso del desarrollo descritos en los siguientes puntos.

\section{Investigación}

La primera etapa que comprende la metodología DCU se abarcó mediante un estudio contextual llevado a cabo en las instalaciones del preescolar Naxhó Café con el objetivo de conocer la situación actual de la lengua materna mazateca desde la opinión de los profesores bilingües.

Como lo señalan los profesores, los trabajos que se han hecho a través de diversas actividades escolares y culturales, padres de familia y la comunidad han contribuido en la conservación de la identidad de todo el pueblo para revalorar, revitalizar y fortalecer la lengua y cultura mazateca. Sin embargo, falta mucho por trabajar y llevar a cabo más acciones que permitan la conservación de identidad cultural y linguística de la comunidad, sobre todo en el aspecto educativo. 


\section{Análisis}

Derivado del estudio contextual se identificó que no existe ningún trabajo previo que vincule el uso de las TIC en la enseñanza de la lengua mazateca variante de Huautla de Jiménez, esto permitió fortalecer más la idea de contribuir en la labor de enseñanza del mazateco empleando las TIC.

\section{Diseño}

Continuando con la siguiente etapa de DCU, y con apoyo de los profesores de Naxhó Café se propusieron dos actividades acordes a las necesidades educativas y al contexto cultural de la educación indígena:

Tarjetas con reconocimiento de marcadores: es un conjunto de 24 tarjetas con representaciones gráficas de animales característicos de la región Cañada, éstos son marcadores que proporcionan información adicional a través de un modelo tridimensional con animación, audio en español, audio en mazateco y su respectivo nombre. A través de prototipos de baja y alta fidelidad se concretó el diseño de las tarjetas, como se muestra en la Figura 2.

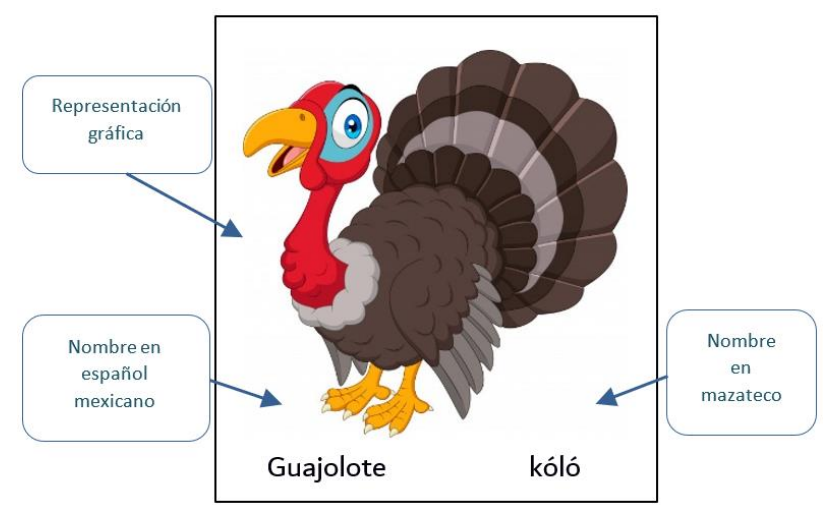

Figura 2 Tarjetas con reconocimiento de marcadores Fuente: Elaboración Propia

Variante del juego de domino de formas empleando el alfabeto mazateco: similar a un juego de mesa de memorama, se diseñaron 53 marcadores que proporcionan el audio en español y audio en mazateco. Del mismo modo, a través de prototipos de baja y alta fidelidad se concretó el diseño de los marcadores de la Figura 3.

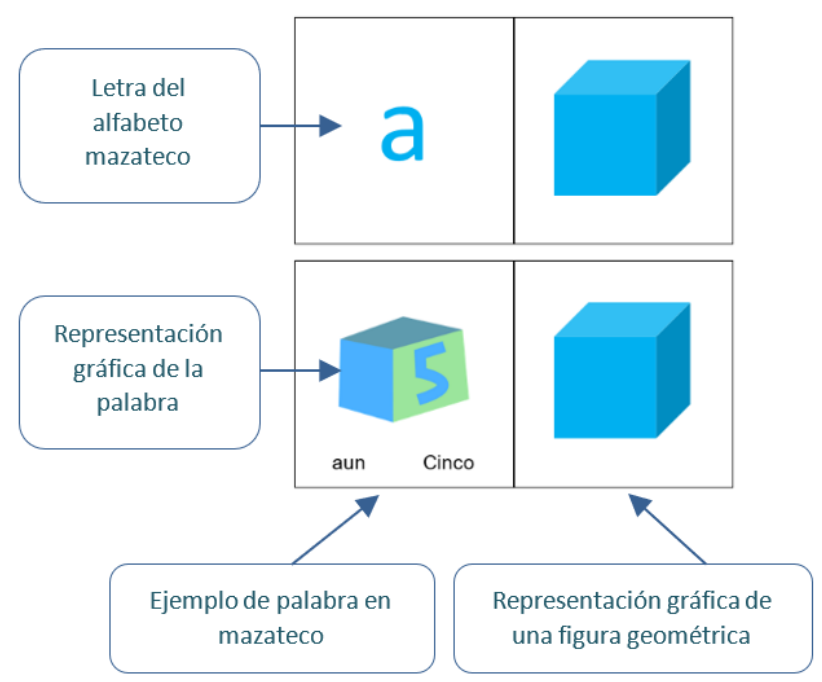

Figura 3 Variante del juego de domino de formas Fuente: Elaboración Propia

Aplicación móvil: un aspecto inherente al desarrollo de cualquier producto o servicio debe ser la usabilidad, que se refiere a la capacidad de un producto en ser entendido, aprendido, usado y atractivo para el usuario, persiguiendo tres aspectos fundamentales:

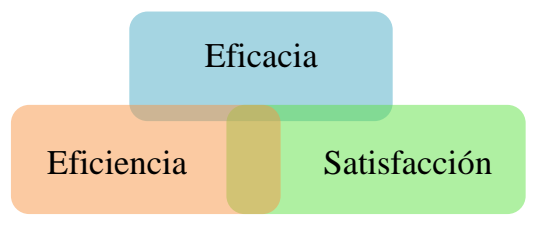

Figura 4 Aspectos de la usabilidad Fuente: Elaboración Propia

Partiendo de los principios de la usabilidad, y con el análisis de la información recopilada se procedió al desarrollo de la aplicación para dispositivos móviles con sistema operativo Android.

A partir de un diseño de baja fidelidad hecho el papel, se realizó un bosquejo de la aplicación móvil (Figura 5) para ser evaluado y mejorado con la retroalimentación de los usuarios en las siguientes fases de la metodología DCU. 


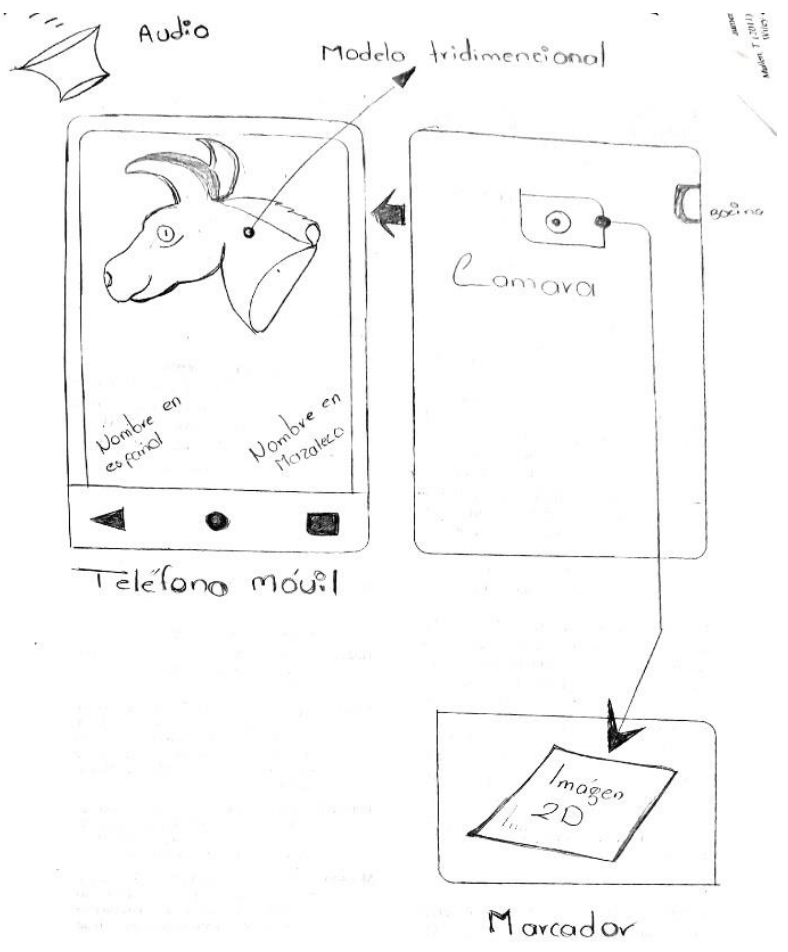

Figura 5 Bosquejo de la aplicación móvil

\section{Evaluación}

En la etapa de evaluación de la aplicación móvil se llevó acabo tres pruebas de usabilidad en las instalaciones de Naxhó Café, en diferentes sesiones con profesores y alumnos de primero, segundo y tercer grado.

Para cada dinámica se asignó diferentes tareas a los participantes, que deberían realizar con los estudiantes a su cargo.

\section{Tarjetas con reconocimiento de marcadores:}

- Tarea 1: enfocar la cámara trasera del dispositivo tecnológico hacia un activador de realidad aumentada.

- $\quad$ Tarea 2: escanear e identificar el objeto 3D mostrado en la aplicación móvil.

- Tarea 3: repetir el sonido emitido de la aplicación móvil.

- Tarea 4: pronunciar en voz alta la palabra en español mexicano y lengua materna mazateca respectivo al ejemplo proporcionado.

\section{Tarjetas con reconocimiento de marcadores:}

- Tarea 1: en un conjunto de 20 tarjetas sobrepuestas en una mesa, el usuario tiene que encontrar los pares iguales y ordenarlos.

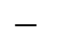

Tarea 2: enfocar la cámara trasera del dispositivo hacia un activador de realidad aumentada.

Tarea 3: pronunciar en voz alta la palabra en español mexicano y lengua materna mazateca respectivo al ejemplo proporcionado.

El objetivo de cada sesión de prueba fue identificar los aspectos de usabilidad obteniendo resultados cualitativos y cuantitativos, además de determinar la satisfacción del participante. De esta manera, en cada iteración las herramientas fueron adaptándose acorde a las necesidades de los profesores.

\section{Adaptación}

Se hicieron tres pruebas de usabilidad, como se mencionó anteriormente. En la primera se proporcionó la herramienta usando una tableta para llevar a cabo la primera actividad (ver Figura 6 y Figura 7).

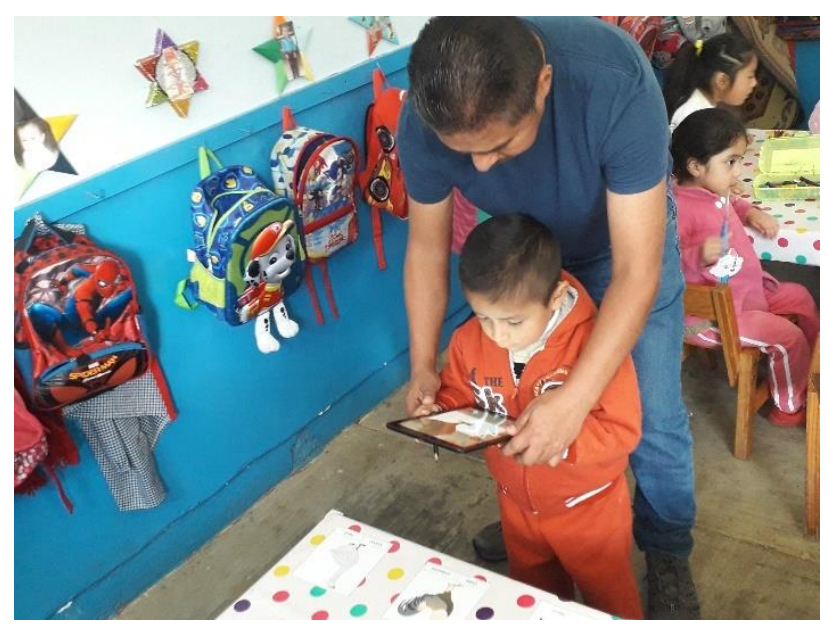

Figura 6 Primera pruebas de usabilidad, empleando las tarjetas con reconocimiento de marcadores

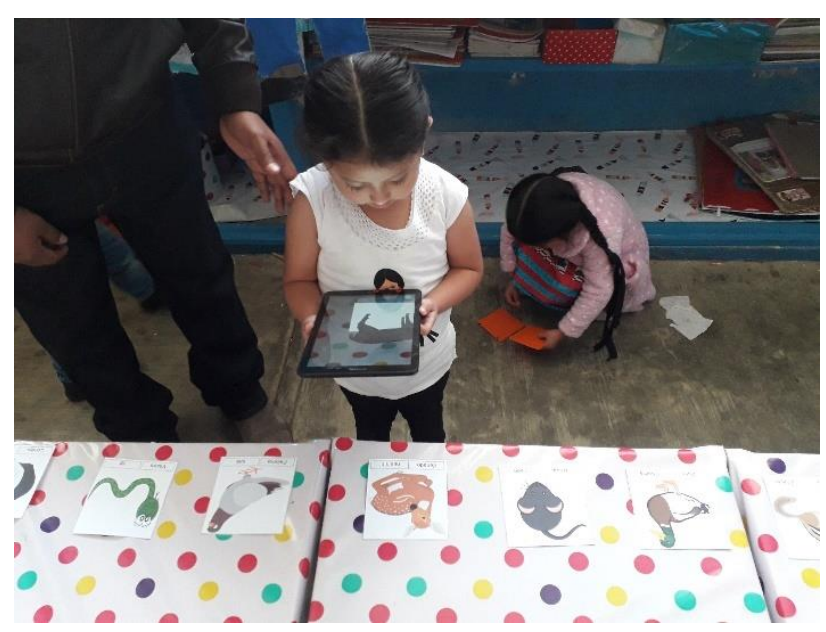

Figura 7 Primera pruebas de usabilidad, empleando las tarjetas con reconocimiento de marcadores

MOTA-CARRERA, Luis Cresencio, MÁRQUEZ-DOMÍNGUEZ, José Alberto, SABINO-MOXO, Beatriz Adriana y SÁNCHEZ-ACEVEDO, Miguel Ángel. Herramienta para la enseñanza de la lengua Mazateca basada en Realidad Aumentada. Revista de Cómputo Aplicado. 2019. 
Las observaciones de la primera prueba son los siguientes:

- $\quad$ La dificultad de manipular la tableta ya que se les hacía pesada a los niños al ir pasando por cada uno de los marcadores.

- $\quad$ Haber utilizado objetos tridimensionales demasiado grandes dificultaba la apreciación completa de cada una.

- Al enfocar la cámara a los marcadores y mostrar los modelos tridimensionales en el dispositivo, se confundía con los colores de fondo.

- $\quad$ El volumen de sonido de la tableta no era tan fuerte para ser escuchada correctamente los audios en un ambiente de ruido.

- Las mejoras que se realizaron en esta sesión fueron las siguientes:

- $\quad$ En lugar de una tableta, se propuso la utilización de un teléfono celular.

- Se colocó un fondo blanco a los objetos tridimensionales para una mejor visualización.

- Se redujeron de tamaño los objetos tridimensionales.

- La utilización de una bocina conectada al dispositivo tecnológico vía bluetooth, para contar con un nivel de volumen mayor (Figura 8).

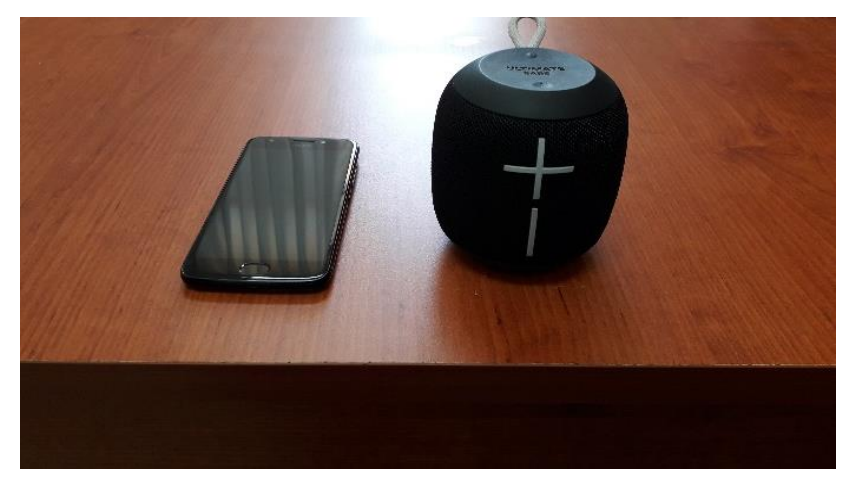

Figura 8 Teléfono celular y bocina bluetooth

En una segunda sesión, realizando los cambios respectivos, la prueba de usabilidad se llevó a cabo mediante un teléfono celular (ver Figura 9 y Figura 10).

El tamaño del teléfono celular hacía mucho más fácil su manipulación, con la reducción de tamaño de los objetos tridimensionales y el fondo blanco se podían apreciar desde el ángulo de visión de los niños.

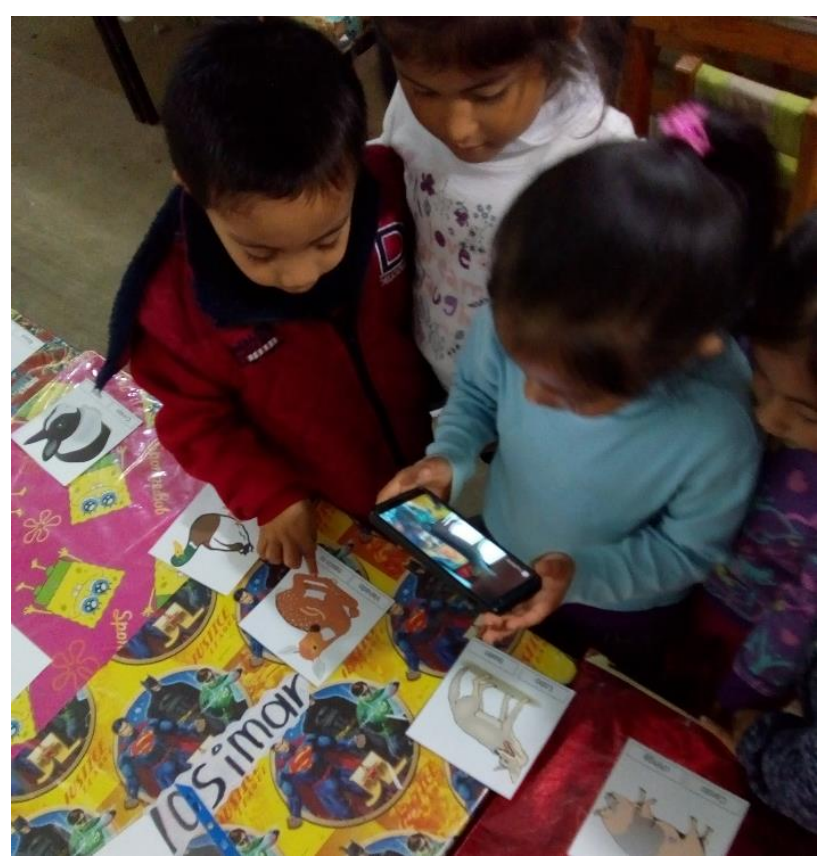

Figura 9 Segunda prueba de usabilidad, empleando las tarjetas con reconocimiento de marcadores

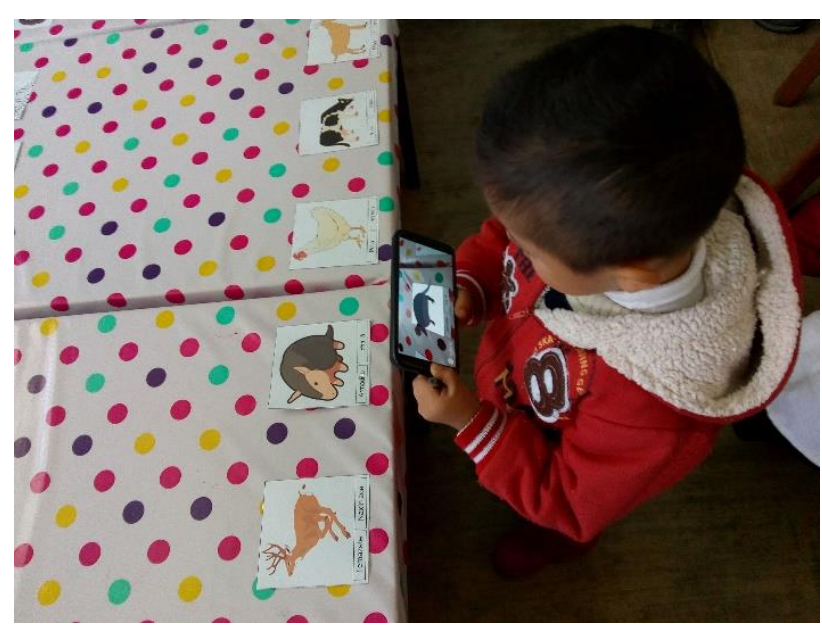

Figura 10 Segunda prueba de usabilidad, empleando las tarjetas con reconocimiento de marcadores

Las observaciones de la segunda prueba son los siguientes:

- La iluminación de los objetos tridimensionales era muy tenue.

- La forma de colocar las tarjetas con reconocimiento de imágenes influía en el aspecto visual de los usuarios para enfocar la cámara del teléfono celular.

- $\quad$ Los profesores sugirieron la posibilidad de ver el nombre del animal en la pantalla del dispositivo tecnológico dentro del espacio en blanco colocado.

- Las mejoras que se realizaron en esta sesión fueron las siguientes:

- Colocar mayor iluminación de los objetos tridimensionales. 
- Utilizar una base para colocar las tarjetas con reconocimiento de marcadores permitiendo mayor comodidad al momento de utilizar la herramienta.

- Y la colocación del nombre en texto tridimensional.

- $\quad$ En la última prueba de usabilidad, ya no se realizaron cambios en la herramienta para llevar a cabo ambas actividades.

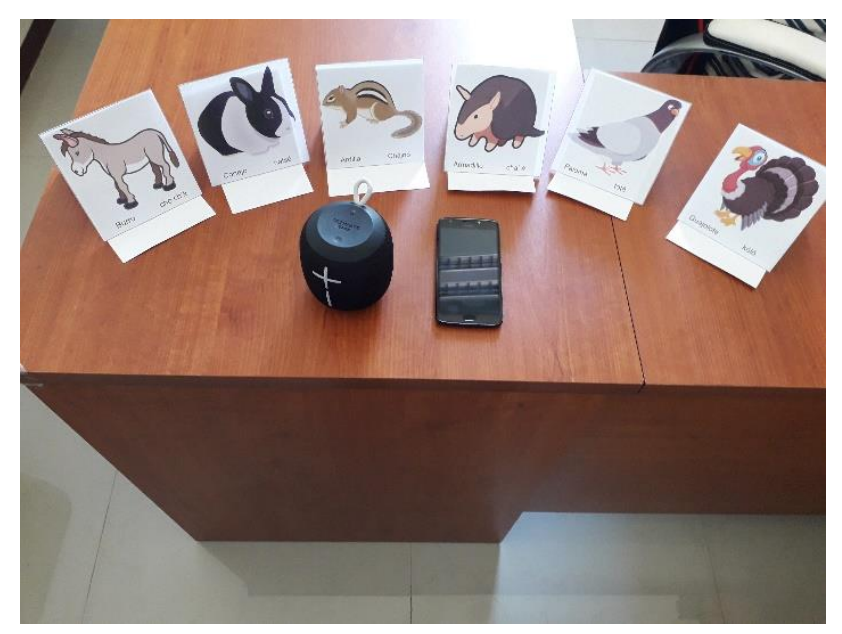

Figura 11 Tarjetas con reconocimiento de marcadores

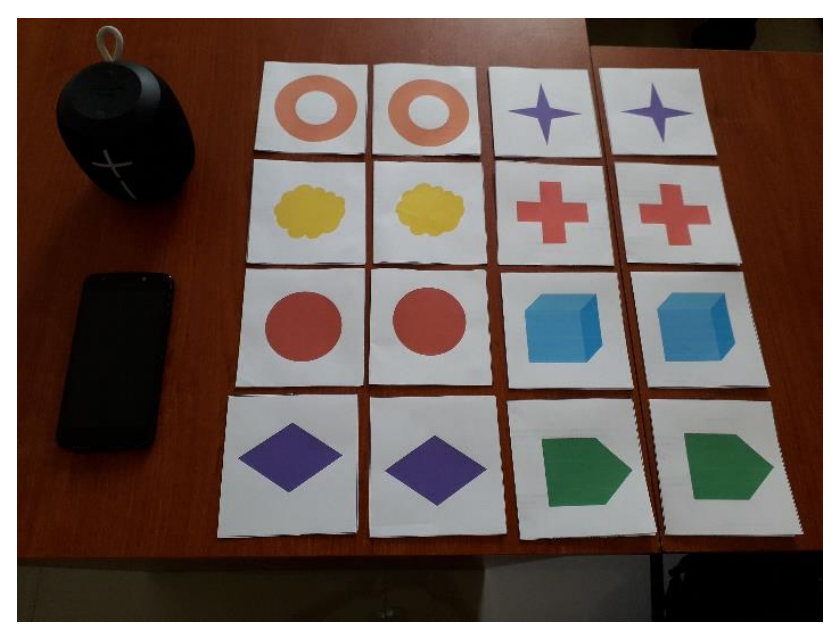

Figura 12 Variante del juego de domino de formas

\section{Resultados}

Recopilando los datos de las tres pruebas de usabilidad, llevada a cabo con profesores y alumnos de primer, segundo y tercer año de preescolar, en la Tabla 1 se muestran los resultados de aplicar la herramienta.

\begin{tabular}{|l|l|}
\hline & Pruebas de usabilidad \\
\hline \multirow{5}{*}{ Eficacia } & $\begin{array}{l}\text { Con el objetivo de saber si la herramienta } \\
\text { cumple con el propósito para la cual fue } \\
\text { diseñada, se midió el desempeño de las } \\
\text { tareas mencionadas en la etapa de } \\
\text { evaluación. } \\
\text { Cumplimiento del 100\% de las tareas } \\
\text { asignadas a profesores y alumnos de la } \\
\text { institución educativa empleando las } \\
\text { herramientas. }\end{array}$ \\
\hline
\end{tabular}

\begin{tabular}{|c|c|}
\hline Eficiencia & $\begin{array}{l}\text { Para conocer el tiempo promedio para llevar } \\
\text { a cabo las tareas mencionadas en la etapa de } \\
\text { evaluación, se midieron en minutos las } \\
\text { actividades a desempeñar por cada usuario. } \\
\text { Un promedio de } 5 \text { minutos por cada } 4 \\
\text { marcadores, utilizado las tarjetas con } \\
\text { reconocimiento de marcadores. } \\
\text { Un promedio de } 5 \text { minutos por cada } 10 \\
\text { marcadores de la variante del juego de } \\
\text { domino de formas. }\end{array}$ \\
\hline Satisfacción & $\begin{array}{l}\text { Al termino de cada actividad, a los usuarios } \\
\text { profesores se les pidió asignar una } \\
\text { calificación a la herramienta utilizada. } \\
\text { En una escala del } 1 \text { al } 10 \text { se obtuvo una } \\
\text { calificación promedio de } 9.5 \text { dada por los } \\
\text { profesores del preescolar acorde a la } \\
\text { utilización de la herramienta. }\end{array}$ \\
\hline
\end{tabular}

Tabla 1 Resultados de las tres pruebas de usabilidad Fuente: Elaboración Propia

Referente a los aspectos de usabilidad con los resultados obtenidos en la Tabla 1, los profesores concluyeron que la herramienta es muy útil ya que abarca una nueva forma de enseñanza utilizando la tecnología de la RA y a los estudiantes se les hace atractivo y fácil de utilizar la herramienta.

\section{Agradecimientos}

A los profesores y alumnos del preescolar Naxhó Café por ser nuestros principales usuarios y colaboradores para llevar a cabo el presente trabajo.

A la licenciada Alejandrina Pedro Castañeda por la traducción de palabras del español al mazateco.

A la ciudadana Elvia Cerqueda Delgado, originaria de Huautla de Jiménez, por ayudarnos en la grabación de las palabras en mazateco y español.

A la Universidad de la Cañada por darme los medios y apoyo para acudir al preescolar y llevar a cabo las pruebas con las herramientas desarrolladas.

\section{Conclusiones}

A través del tratamiento informático de la lengua mazateca variante de Huautla de Jiménez para su almacenamiento, procesamiento y presentación con medios electrónicos como lo son los dispositivos móviles, se hace posible la documentación y conservación a través del tiempo. 
Como lo indican los profesores del preescolar, la herramienta que se les proporciono es de gran ayuda, ya que a los niños se les hace muy interesante y entretenido, a su vez, los diversos contenidos que se integraron coadyuvan en las dinámicas de enseñanza de la lengua Mazateca impartida por los educadores. Con lo anterior se puede deducir que un correcto enfoque y empleo de las TIC en el área educativa trae consigo grandes beneficios, más aún con el rescate de la lengua Mazateca.

Con el proyecto desarrollado se obtuvo una herramienta tecnológica que sirve para la enseñanza de la lengua materna mazateca y que es empleada en el centro de educación preescolar Naxhó Café, acercando a los niños al uso de la tecnología a través de la educación en el primer nivel de estudio escolar, a través de una educación supervisada y controlado por el docente.

\section{Referencias}

Blender (2019). Recuperado de: https://www.blender.org/about/

Carrera G. C. (2011). Acercamiento gramatical a la lengua mazateca de Mazatlán Villa de Flores, Oaxaca. México: Talleres Gráficos de México. Recuperado de: https://site.inali.gob.mx/pdf/libro_gramatica_m azateca.pdf

Galdames V., Walqui A. \& Gustafson B. (2006). Enseñanza de lengua indígena como lengua materna. Bolivia: Editorial. Recuperado de: https://eib.sep.gob.mx/isbn/dl41105605.pdf

Garreta D, M \& Mor P, E (2019). Diseño centrado en el usuario. PID_00176058. Recuperado de: https://www.exabyteinformatica.com/uoc/Infor matica/Interaccion_persona_ordenador/Interacc ion_persona_ordenador_(Modulo_3).pdf

Guzmán C, C. (2011). Acercamiento gramatical a la lengua mazateca de Mazatlán de Villa de Flores, Oaxaca. INALI. Recuperado de: https://site.inali.gob.mx/pdf/libro_gramatica_m azateca.pdf
INALI - Instituto Nacional de Lenguas Indígenas. (2015). Proyecto de indicadores sociolingüísticos de las lenguas indígenas nacionales. Recuperado de: https://site.inali.gob.mx/Micrositios/estadistica basica/estadisticas2015/pdf/general/general7.pd $\mathrm{f}$

INPI - Instituto Nacional de los Pueblos Indígenas (2017). Etnografía del pueblo mazateco de Oaxaca - Ha shuta Enima. https://www.gob.mx/inpi/articulos/etnografiadel-pueblo-mazateco-de-oaxaca-ha-shuta-enima PROINALI - Programa Institucional del Instituto Nacional de Lenguas Indígenas (2014). Recuperado de: https://site.inali.gob.mx/publicaciones/Proinali_ 2014.pdf

Programa Especial de los Pueblos Indígenas (2014 - 2018). Recuperado de: https://www.gob.mx/cms/uploads/attachment/fi le/32305/cdi-programa-especial-pueblosindigenas-2014-2018.pdf

$\begin{array}{lll}\text { Pixabay (2019). } & \text { Recuperado de: } \\ \text { https://pixabay.com/ } & & \\ \text { Poly-Google (2019). } & \text { Recuperado de: } \\ \text { https://poly.google.com/ } & & \end{array}$

Rigueros B, C. (2017). La realidad aumentada: lo que debemos conocer. Tecnología Investigación y Academia, 5(2), 257-261. Recuperado de https://revistas.udistrital.edu.co/ojs/index.php/ti a/article/view/11278

Secretaría de Cultura. (21 de febrero de 2018). ¿Sabías que en México hay 68 lenguas indígenas, además del español? [Mensaje en un blog]. Recuperado de: https://www.gob.mx/cultura/articulos/lenguasindigenas?idiom $=\mathrm{es}$

Unity 3D (2019). Recuperado de: https://unity.com/solutions/mobile-ar Vuforia Engine (2019). Recuperado de: https://engine.vuforia.com/engine 